\title{
Simulation, visualization and dosimetric validation of scatter radiation distribution under fluoroscopy settings
}

\author{
Lawrence WC Chan ${ }^{1}$, TP Chan ${ }^{1}$, Ben TF Cheung ${ }^{1}$, Kelvin Mo ${ }^{2}$, Karl KL Fung ${ }^{1}$ \\ 1. Department of Health Technology and Informatics, Hong Kong Polytechnic University, Hong Kong. 2. Radiology \\ Department, Union Hospital, Hong Kong.
}

Correspondence: Lawrence WC Chan. Address: Department of Health Technology and Informatics, Y902, 9/F, Lee Shau Kee Building, Hong Kong Polytechnic University, Hung Hom, Kowloon, Hong Kong. Email: wing.chi.chan@polyu.edu.hk

Received: June 24, 2015

DOI : $10.5430 /$ jbei.v1n1p93
Accepted: August 10, 2015

URL: http://dx.doi.org/10.5430/jbei.v1n1p93

\section{Abstract}

Objective: This study developed a three-dimensional visualization method for presenting the geometric pattern of the relative dose generated by Monte Carlo (MC) simulation and validated the simulated dose values against the physical measurement.

Methods: In fluoroscopy and interventional radiology examinations, relatively high level of occupational dose is delivered to healthcare workers due to prolonged radiation exposure in imaging procedures. As the spatial difference in dose is never negligible, the scatter radiation distribution under fluoroscopic exposures is thus worth investigating. MC simulation is a sophisticated statistical method, which has been widely applied for modeling scatter radiation in general $\mathrm{X}$-ray examination room. However, the application and validation of MC simulation under fluoroscopy setting have not been found yet. In this study, a stack of tissue equivalent slabs were used as the object under fluoroscopic irradiation. EGSnrc-based DOSXYZnrc code was applied to simulate the scatter dose distribution and the physical measurement of radiation dose was performed using an ionization chamber radiation detector.

Results: At 19 representative locations taking into account the work area and radiosensitive organs of healthcare workers, the trend compliance of the simulated with the measured dose values was examined using the correlation analysis. A significant monotonic association between the MC simulation and physical measurement of dose values was identified $\left(r_{s}=0.822\right.$ and $\left.P<.01\right)$. At the same horizontal distance from the irradiation axis, it was observed that the air dose was relatively higher at the level of gonad region than at the eye level.

Conclusions: The proposed visualization approach illustrates a three dimensional dose simulation in local display density, which arouses the awareness about prolonged radiation exposure in clinical environment. The reliability and validity of the simulation were examined.

\section{Key words}

Monte Carlo simulation, Fluoroscopy, Radiation dosimetry, Homogeneous slabs, 3D visualization 


\section{Introduction}

\subsection{Clinical applications of fluoroscopy and its radiation dose}

Fluoroscopy examinations have become more common in diagnostic radiology since the $20^{\text {th }}$ century due to the wide application of interventional and contrast studies to functionally study the human anatomy and assist the treatment of pathologies ${ }^{[1,2]}$. However, the occupational dose was found the highest during the procedures involving the use of fluoroscopy units as shown in previous research studies ${ }^{[3,4]}$. For instance, common fluoroscopy examinations including barium studies like barium enema and meal studies contribute to about $25 \%$ of the collective effective dose from man-made sources ${ }^{[5]}$. Besides, International Atomic Energy Agency (IAEA) stated that the patient mean effective dose in a fluoroscopy examination was found to be 100 to 390 times more than a routine postero-anterior chest X-ray examination and the UNSCEAR 2,000 report stated that fluoroscopic procedures were the major source of radiation dose to clinical staff in medicine ${ }^{[6]}$. Damages to eye lens and other body parts of medical staff are possibly induced by radiation after working in the fluoroscopy department for a few years unless adequate radiation protection is provided ${ }^{[7,8]}$. Therefore, International Commission on Radiological Protection (ICRP) and National Radiological Protection Board (NRPB) have published recommendations and research on radiation protection methods and suggesting standards, especially for the advanced and complex examinations which deliver high radiation dose to staff, in order to give advice to healthcare workers to well protect themselves from radiation ${ }^{[9]}$.

The radiation dose delivered to the staff mainly originates from the scatter radiation. The knowledge on the nature and geometric patterns of scattered radiation has long been inadequate and researchers have proposed different methods to identify the scattering properties based on different radiation sources. General X-ray machines, computed tomography and radionuclide imaging have been considered as the main foci in radiology studies ${ }^{[10-15]}$. However, study focusing on fluoroscopy has not been found yet. Previous researches were concerned about the impact of scattered radiation on patient dose $^{[10,11,14,16,17]}$ and radiographic image quality ${ }^{[14,18,19]}$, and the physical properties of radiation ${ }^{[12,20]}$, but there has not been study on the occupational dose of radiology department staff. Besides, the results obtained in general X-ray study cannot represent validly in fluoroscopy because the properties of these two modalities are different. Fluoroscopy units use continuous pulsating radiation output and general X-ray machines delivers one-off radiation in time period of milliseconds. Besides, the internal structures, like anode angle selection, and the position of the X-ray tube are different in the two modalities.

\subsection{Physical measurement tools}

To precisely measure radiation dose physically, equipment like dose-area-product (DAP) meter, ionization chamber or thermo luminescent dosimeters (TLD) can be used ${ }^{[21]}$. The DAP meter ensures the consistency of the exposure output for scattering measurement. TLD can provide reading of scattered dose but there are several concerns over its feasibility in research studies. As each TLD pellet can only produce a reading after measurement, it is very time consuming for dose reading and annealing process in order to allow the re-use of the pellet for multiple measurements. Besides, the TLD is not sensitive enough to detect the low scatter air dose below the level detectable by TLD reading system. Since the ionization chamber has been considered a gold standard in measuring scattering in diagnostic radiology and can produce instantaneous and accurate dose readings ${ }^{[22,23]}$, it was properly used for massive parallel measurement of dose.

\subsection{Monte Carlo simulation in radiology studies}

Many research studies have been performed to investigate the radiation protection methods but the depth and significance of these studies were limited by medical ethical issues with respect to human rights ${ }^{[24]}$. It is because a research study putting human or animal subjects at risk of unnecessary radiation exposure violates the Declaration of Helsinki, a formal ethical guideline recognized by national legislation and World Health Organization ${ }^{[25]}$. Even if physical measurement could be taken in an 'ethical' way, the results would not be totally convincible due to the experimental design with numerous assumptions made, possible analytic error and errors from instruments. Besides, it would be difficult to take 
readings at all possible locations within the work area of an examination room. Until the development of MC simulation techniques, comprehensive dosimetric study was thought to be impossible, dangerous or unethical ${ }^{[26]}$. Therefore, there is a need to adopt and validate the MC simulation code in radiology study in order to unveil its potential for testifying more radiation protection measures for healthcare workers.

MC simulation, which was first applied and experimented in Buffon's needle test in 1930s ${ }^{[27]}$, involves the use of a series of mathematical equations under physics and probability theories. It relies on repeated random sampling to produce results using different computational algorithms. MC simulation methods include three major applications: optimization, numerical integration and generation of samples from a probability distribution. MC simulation in radiology studies numerically models photon transport according to radiative transfer equation (Boltzmann equation), which depicts the photon motion in biological tissues. Different outcomes can be obtained in a flexible way by changing any of the physical parameters, including exposure factors, incident beam energy, photon quantity and scattering medium. Thus, simulation can be applied not only for estimating the spatial patterns of scatter radiation ${ }^{[18]}$, but also for studying the contrast, noise, dose and grid performance ${ }^{[28,29]}$. In comparison with physical measurement approach, MC simulation provides more detailed dose information in cost-effective way ${ }^{[30]}$. Some codes, like EGS4 (Electron-Gamma Shower $4^{\text {th }}$ version) and SIERRA, were developed to simulate the photon transport and scattering ${ }^{[27,31]}$. As MC simulation involves plenty of branching and inter-relating equations and complex codes, there are doubts about its reliability and accuracy, demanding systematic validation.

\subsection{Three-dimensional visualization}

Computerized visualization makes human-unperceivable scatter radiation visible. A study presented an approach to visualize the simulated scatter radiation in a training system for mobile image intensifier ${ }^{[32]}$. Although the system was evaluated through questionnaire survey, there was no physical dose measurement for validating the rendered distribution. Therefore, the use of the system was limited to education purposes only. Another study proposed a device for predicting the scatter radiation based on the instantly measured values at particular locations and displaying two-dimensional (2D) contours and three-dimensional (3D) distributions of scatter radiation ${ }^{[33]}$. However, the $2 \mathrm{D}$ display presents the vertically projected contours of scatter radiation with equal intensities and the 3D display depicts the scatter X-ray paths in pencil beams. This study is aimed to develop a visualization technique for presenting the simulated dose values in virtual 3D scene.

\section{Methods}

\subsection{Physical measurement instrumentation}

Measurement was carried out in the fluoroscopy examination room at the Union Hospital, Hong Kong. The fluoroscopy unit used was GE Medical INNOVA 4100-IQ model with an under-couch X-ray tube with target angle of $11.5^{\circ}$. Yearly quality assurance tests on the physical functioning and the consistence of radiation output were performed by service engineer. Besides, daily operational quality assurance tests with a standard aluminum phantom were conducted by the operators.

A calibrated ionization chamber radiation detection system ("Radcal 9015", California, USA) connected with a $180 \mathrm{~cm}^{3}$ chamber was used for the measurement of air dose. Back-scattered radiation was kept to the minimum by application of 1 $\mathrm{mm}$ lead equivalent lead rubber sheets over the likely scattering media, such as the edge of the X-ray table.

Twenty pieces of $30 \mathrm{~cm} \times 30 \mathrm{~cm} \times 1 \mathrm{~cm}$ (length, width and thickness respectively) tissue equivalent slabs (PTW Co, Freiburg, Germany) were used in this study. They are homogeneous in density and tissue-equivalent. By attenuation tests on radiation exposure, 20 pieces of dry slabs was found in the attenuation ability to be equivalent to the abdomen of an 
adult anthropomorphic phantom. For simplicity, the amount and pattern of scatter radiation so produced was assumed to be similar to that of an adult abdominal fluoroscopic screening examination.

\subsection{Physical measurement setup}

The stack of slabs was placed onto the X-ray couch in alignment with the axes of the couch. The central vertical axis was placed aligning with the center of the flat panel detector of the fluoroscopy unit through preliminary screening by fluoroscopy unit and positioning adjustment. The focus-to-object distance (FOD) will be maximized to $74 \mathrm{~cm}$ and the flat panel detector was positioned at minimal distance with the upper edge of slabs to simulate the real fluoroscopy examination condition.

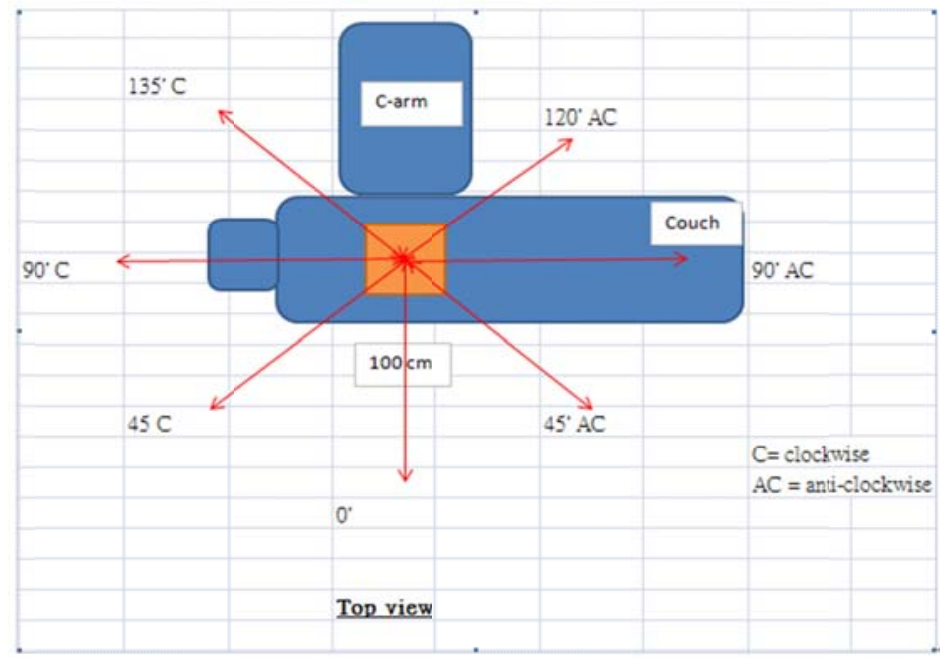

Figure 1. Top view of the measurement set-up showing the locations of ionization chamber at different angles

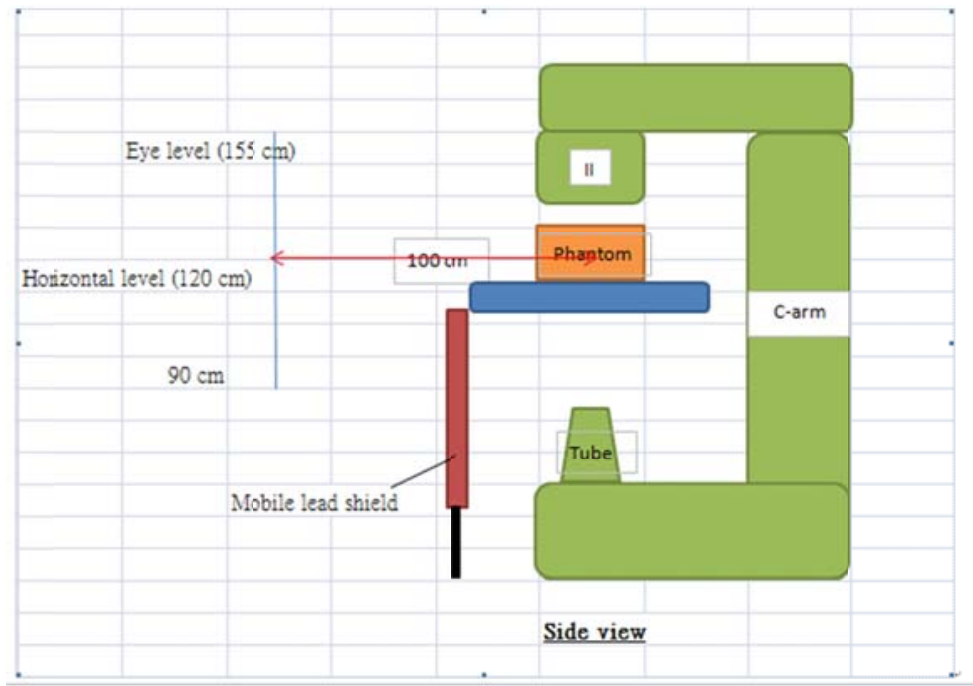

Figure 2. Side view of the measurement setup showing the locations of ionization chamber 3 levels, $155 \mathrm{~cm}, 120 \mathrm{~cm}$ and $90 \mathrm{~cm}$ at each angle. Mobile lead shield was placed near the tube.

Figures 1 and 2 show the top-view and side-view of the physical measurement setup. The heights of measuring points considered in this study include gonad level at $90 \mathrm{~cm}$ from the ground, level horizontal to the mid-level of the slab stack at $120 \mathrm{~cm}$ and eye level at $150 \mathrm{~cm}$. The levels of $155 \mathrm{~cm}$ and $90 \mathrm{~cm}$ were chosen because radiosensitive organs, including 
lens and reproductive organs, are likely to be found at these levels ${ }^{[34]}$. At each height, air dose readings using the ionization chamber were taken at different locations with distance $100 \mathrm{~cm}$ distant from the center of the stack and each at $45^{\circ}$ apart towards the center direction. The fluoroscopy settings could not allow the measurement at $180^{\circ}$ at all levels, at $90^{\circ}$ anti-clockwise (AC) and $120^{\circ} \mathrm{AC}$ at gonad level because the bulk of the c-arm and the couch hindered the positioning of the ionization chamber at these locations. Ionization chamber was fixed with clamp and stand for stabilization during all measurements. And a mobile lead shield was placed closed to the X-ray tube at the side near the ionization chamber to minimize possible leakage and scatter radiation from the X-ray tube and other scattering media such as the X-ray couch. Exposure factors were set at $80 \mathrm{kVp}$ and $5.6 \mathrm{mAs}$ and the field of view (FOV) $40 \mathrm{~cm} \times 40 \mathrm{~cm}$. The protocol set was "General Abdomen" and the exposures were taken using "Image Acquisition Mode". Ten readings were taken at each feasible location.

\subsection{Monte Carlo simulation}

Electron gamma shower (EGS) is a package of code system for MC simulation of coupled transport of electrons and photons with available energy range from $1 \mathrm{KeV}$ to $10 \mathrm{GeV}$ and this has been widely applied in diagnostic radiology and radiotherapy studies. The simulated photons are transported in steps of random length. EGSnrc is an updated version of EGS4 with improvement and extension in simulating charged particle transport and low energy cross-sections. EGSnrc code system takes into account the diagnostic energy range involving several radiation interaction processes, such as Bremsstrahlung production, positron annihilation, pair production, Compton scattering, coherent Rayleigh scattering and photoelectric effect. All these interaction processes are considered in the dose calculation. The probability of different types of particle interaction and their amounts of energy transfer are determined by the EGSnrc code system based on the simulated energy range and the properties of the interacting medium ${ }^{[35]}$.

The DOSXYZnrc code is an EGSnrc-based code applied for simulating the X-ray units and calculating the radiation dose in and out of the medium. Graphic user interface (GUI) of DOSXYZnrc facilitates parameter input and interacting medium specification. The medium can be specified using numerical values of Cartesian coordinates or three-dimensional image data of voxels. External files describing the X-ray spectrum and medium geometry and compositions can be read by EGSnrc code system. Since the particle interaction is probabilistic, random number generator (RNG) seeds are used to randomly decide which interaction process or event will occur. In the simulation, the scatter air dose is calculated in terms of air kerma in its located voxel. The scatter air kerma is calculated based on the fluence with the scattered photon energy and the mass energy transfer coefficient ${ }^{[35-37]}$.

To simulate the photon transport, the MC simulation program first launches photon packets by defining the initial position and direction of movement of photons, as shown in Figure 3. The step size, which is the distance travelled between defined media, is then determined. Within the media, absorption and scattering occur and the energy and the movement direction of the photons are changed. If the energy of the transmitted photons is below the pre-defined photon cut-off energy, the photons are considered terminated. On the other hand, if the energy of the transmitted photon is not below the cut-off energy, the photons will continue to interact with the media according to their newly defined position and direction, until its energy falls below cut-off energy.

To start the simulation, a new default input file was created in the DOSXYZnrc GUI. Two media, the air and slabs, were modeled by the preparation code of EGS4, PEGS4 code. A point source with rectangular collimation was incident toward the slabs from the bottom. The $80 \mathrm{kVp} \mathrm{X}$-ray spectrum showing the relative number of photons at different photon energy was imported into the program to simulate the probabilistic quantity of photons at the time of X-ray emission. Implementation of 108 histories controls the error values of the simulated scatter dose within an acceptable range. A $3 \mathrm{~d}$ dose file was generated after the completion of simulation. The simulated dose values at the locations of physical measurement were read using Matlab. Simulation was performed for three times with different random number generator seeds. The average of repeatedly simulated dose values at each location was considered for further analysis. 


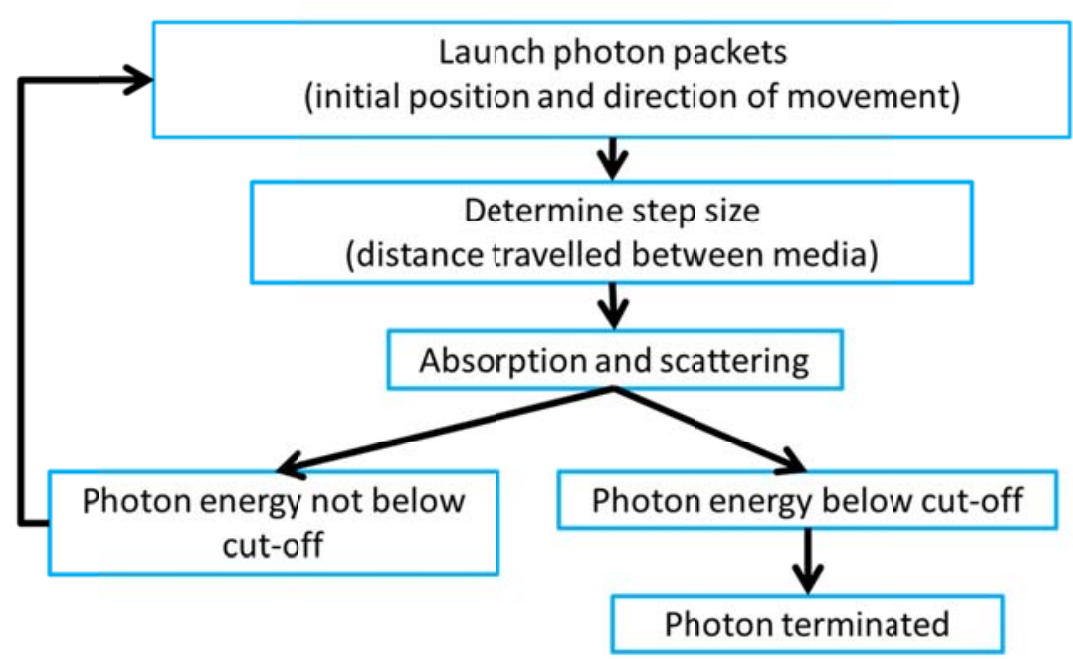

Figure 3. The brief flow of photon transport simulation by MC program

\subsection{Statistical analysis}

Shapiro-Wilk (SW) normality test was performed to check if the measured and simulated dose values follow normal distribution. As it is shown in the next section that the simulated dose values were not normally distributed, Spearman's correlation test, a non-parametric test, was performed to identify the monotonic association between simulated and measured dose values at the considered locations. IBM SPSS software was used for the tests.

\subsection{D visualization}

After MC simulation process, the simulated dose values in a 3D array of voxels were set as $3 \mathrm{~d}$ dose file format, in which the dimensions and grid points of the array is specified and the voxels are encoded in linear sequence of dose values and errors. Matlab was used to read the file, form a 3D matrix of dose values and construct the corresponding 3D plot. Surface and volume rendering techniques were not considered for the visualization because it is difficult to show the spatial changes in dose values on 3D display of these techniques. Therefore, we developed a visualization technique, in which a dose value is denoted by the density of virtual particles in its voxel. The generation of these virtual particles involves three steps. First, each voxel was divided into $10 \times 10 \times 10$ sub-voxels. Second, each dose value, $D$, is linearly transformed to the number of virtual particles, $n$, using the following formula.

$$
N=\text { integer }\left(\frac{D \times N_{\max }}{D_{\max }}\right)
$$

Where $D_{\max }$ is the maximum value of the 3D matrix, $N_{\max }$ is the user-defined upper limit of virtual particle number. Finally, $N$ sub-voxels are selected randomly and filled to represent the virtual particles in each voxel. The dose values can be perceived in terms of densities rather than intensities in $3 \mathrm{D}$ visualization.

\section{Results}

\subsection{Physical measurement results}

At each of the 19 locations of interest, the air dose was measured repeatedly for 10 times. Average measured dose values are summarized in Table 1. The normality assumption cannot be rejected as the SW test indicated $P>.05$. 
Table 1. Dose values obtained from MC simulation and physical measurement, and SW test results

\begin{tabular}{|c|c|c|c|}
\hline Level (cm) & Angle (degree) & Average measured dose $(\mathrm{nGy}) \pm \mathrm{SD}$ & Simulated dose $\times 10^{16}(\mathrm{mGy})$ \\
\hline \multirow[t]{5}{*}{90} & 0 & $770 \pm 6$ & 3.480 \\
\hline & 45 & $747 \pm 7$ & 3.529 \\
\hline & 90 & $704 \pm 6$ & 3.333 \\
\hline & 135 & $690 \pm 5$ & 3.642 \\
\hline & 315 & $740 \pm 6$ & 3.525 \\
\hline \multirow[t]{7}{*}{120} & 0 & $619 \pm 5$ & 2.397 \\
\hline & 45 & $646 \pm 4$ & 2.599 \\
\hline & 90 & $625 \pm 5$ & 2.563 \\
\hline & 135 & $647 \pm 5$ & 2.449 \\
\hline & 240 & $718 \pm 6$ & 2.312 \\
\hline & 270 & $550 \pm 3$ & 2.340 \\
\hline & 315 & $627 \pm 5$ & 2.620 \\
\hline \multirow[t]{7}{*}{155} & 0 & $494 \pm 3$ & 2.007 \\
\hline & 45 & $529 \pm 4$ & 2.332 \\
\hline & 90 & $520 \pm 3$ & 2.139 \\
\hline & 135 & $520 \pm 4$ & 2.384 \\
\hline & 240 & $506 \pm 4$ & 2.014 \\
\hline & 270 & $520 \pm 4$ & 2.148 \\
\hline & 315 & $541 \pm 4$ & 2.357 \\
\hline \multirow[t]{2}{*}{ SW test } & Statistic W & 0.910 & 0.825 \\
\hline & $P$-value & 0.073 & 0.003 \\
\hline
\end{tabular}

\subsection{MC simulation results}

A 3D array with dimensions $25 \times 25 \times 14$ was simulated to model the dose distribution in a space of $250 \mathrm{~cm} \times 250 \mathrm{~cm} \times$ $140 \mathrm{~cm}$ in size. The simulation was performed with the error controlled below $10 \%$. The simulation process was repeated for 3 times with difference RNG seeds. The average simulated dose values over the 19 locations of interest were shown in Table 1. The SW test indicated non-normal distribution with $P<.01$.

\subsection{Correlation test}

Non-parametric test was used to examine the monotonic association between the measured and simulated dose values. Significant association was identified as Spearman's correlation coefficient was found to be $0.822(P<.01)$.

\subsection{Dose visualization}

The 3D dose matrix was further processed and a 3D-plot of dose distribution was constructed, as shown in Figure 4. It was observed that the virtual particles were denser at the lower level than the higher level. 


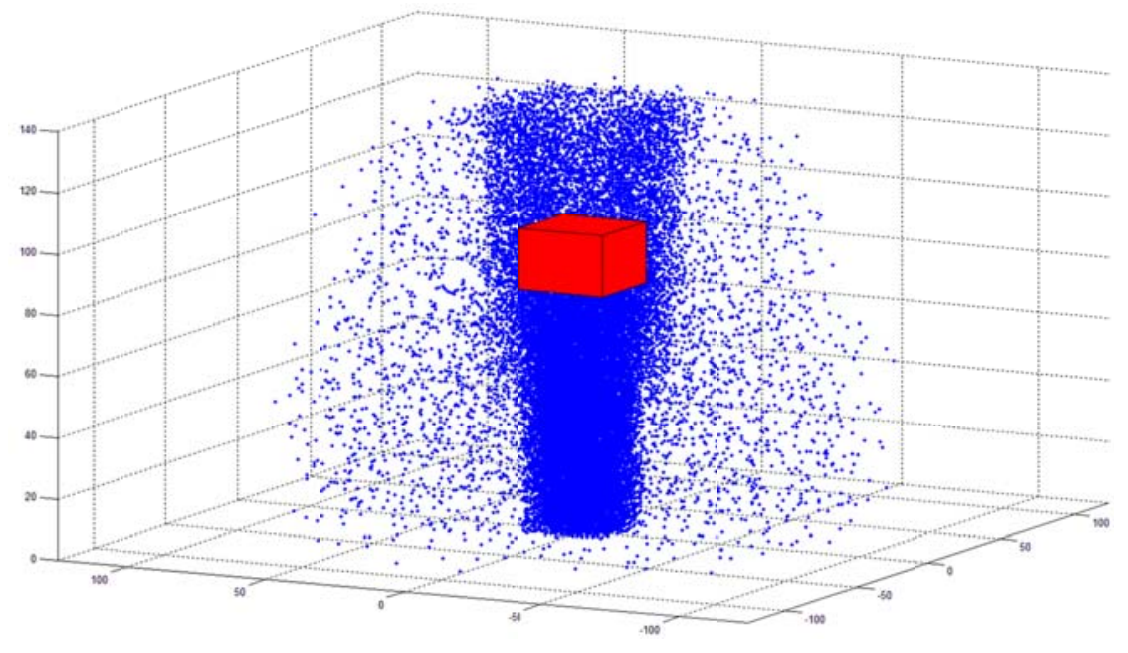

Figure 4. A 3D-plot showing the scatter dose distribution. The radiation source is located at $(0,0,0)$ and the homogeneous slabs are represented by the red block.

\section{Discussion}

\subsection{Clinical application of MC simulation and 3D visualization}

In this study, the results of scattered radiation from homogeneous slabs under fluoroscopic exposure obtained from physical measurement and MC simulation showed significant correlation. The compliance between the measurement and simulation attained by such simple model implicates that more complex model can be simulated. Thus, the DOSXYZnrc is applicable in studying the scattering distribution under fluoroscopic exposures.

It is possible to apply MC simulation method using DOSXYZnrc code to predict the scattered dose within the fluoroscopy room by comparing the measured dose values using ionization chamber at few points for validation. If the trend at these points shows association, comprehensive MC simulation can be performed to calculate the $3 \mathrm{D}$ dose in the room within a reasonable period of time in order to determine the safe work positions for healthcare workers with significantly lower scatter radiation for radiation protection purpose. The 3D dose can be further processed and form a 3D-plot using Matlab in order to visualize air dose in terms of virtual particles.

By incorporating the radiation protection equipment in voxels, the simulated dose distribution is also useful to determine the effectiveness of equipment, such as lead shielding and lead glass, in protecting the staff from radiation.

\subsection{Limitations of dosimetric validation}

However, there are some limitations in the validation regarding the data accuracy. There is inevitable positioning error of ionization chamber with very minor discrepancy as compared with the specified voxel in the simulation. Minor shifting of ionization chamber position due to human error would be possible to affect the compliance of measurement with the simulated results. Also, as the detectable surface of $180 \mathrm{cc}$ ionization chamber is circular in shape over a plane, any minor rotation or tilting or the ionization chamber would result in reduced detectable surface area although in the study, every effort was made to set the maximum detection surface facing the center of the slabs stack. Further, the ionization chamber has low sensitivity in detecting the very low level of scatter radiation intensity Even though the low intensity level of scatter radiation can cause ionization of air within the chamber, the current induced is too tiny to be measured accurately ${ }^{[22]}$. On the contrary, the MC simulation can calculate the possible radiation at all levels of intensity and from different interaction in medium to contribute to the final calculated dose at a specific location. It may be possible to use a 
pressurized ion chamber radiation detection system to increase the current produced and thus sensitivity of measurement ${ }^{[22]}$.

In the physical measurement, devices and associated accessories might affect in a small extent the total amount of scatter radiation to be accurately measured, such as the built-in dose-area-product (DAP) meter at the output of the X-ray tube and ionization chamber support. However, in the MC simulation, it would be difficulty to have exactly similar simulation set up as in the real experiment for physical measurements.

\subsection{Suggested further studies}

It is possible to use the Rando phantom as a better model of human body but its shape and structure are more complex than the slabs. The head component of the Rando phantom can be neglected due to relatively insignificant scattering ${ }^{[38]}$. With the use of program like "ctcreate", Computed Tomography (CT) data sets can be imported to DOSXYZnrc code and the $\mathrm{CT}$ numbers or densities of components of the scanned medium can be considered for MC simulation. Thus, the scatter from the Rando phantom or the actual human body can be studied through simulation.

\section{Conclusion}

EGSnrc-based DOSXYZnrc code was found applicable in simulating a simple model under fluoroscopic exposures in the examination room. Thus, DOSXYZnrc code has the potential in simulating more complex objects, like humanoid phantom, which well simulates the human anatomy for more accurate dosimetric survey. However, the resolution of the simulated environment, i.e. the number of voxels, has to be increased to incorporate such complicated object structure. More precise modeling of X-ray unit and experiment environment should be considered to demonstrate the scatter distribution for establishing radiation protection guidelines purpose. Further, a 3D visualization technique was developed in this study to present the dose distribution intuitively.

\section{References}

[1] Mahesh M. Fluoroscopy: patient radiation exposure issues. Radiographics. 2001; 21(4): 1033-45. PMid:11452079 http://dx.doi.org/10.1148/radiographics.21.4.g01j1271033

[2] Gyekye PK, Schandorf C, Boadu M, et al. Patient dose assessment due to fluoroscopic exposure for some selected fluoroscopic procedures in Ghana. CORD Conference Proceedings. 2009; 136: 203. http://dx.doi.org/10.1093/rpd/ncp172

[3] Valentin J. Avoidance of radiation injuries from medical interventional procedures. Ann ICRP. 2000; 30(2).

[4] ICRP, Radiological protection in medicine. Ann ICRP. 2007; 37(6).

[5] Hughes JS, O'Riordan MC. Radiation Exposure of the UK Population. 1993 Review (National Radiation Protection Board Report R263), (HMSO, London) (1993).

[6] United Nations Scientific Committee on the Effects of Atomic Radiation. Sources and effects of ionizing radiation. UNSCEAR 2000 report to the General Assembly, with scientific annexes. Annex E. Occupational radiation exposures. New York, United Nations. 2000.

[7] Vano E, Kleiman NJ, Duran A, et al. Radiation cataract risk in interventional cardiology personnel. Radiat. Res. 2010; 174(4): 490-5. PMid:20726724. http://dx.doi.org/10.1667/RR2207.1

[8] Ciraj-Bjelac O, Rehani MM, Sim KH, et al. Risk for radiation induced cataract for staff in interventional cardiology: is there a reason for concern? Cathet Cardiovasc Intervent. 2010; 76(6).

[9] Vano E, Gonzalez L, Fernandez JM, et al. Occupational radiation doses in interventional cardiology: a 15-year follow-up. British Journal of Radiology. 2006; 79: 383-8. PMid:16632618. http://dx.doi.org/10.1259/bjr/26829723

[10] Rodrigues P, Trindade A, Peralta L, et al. Application of GEANT4 radiation transport toolkit to dose calculations in anthropomorphic phantoms. Applied Radiation and Isotopes. 2004; 61: 1451-61. PMid:15388147. http://dx.doi.org/10.1016/j.apradiso.2004.05.073

[11] Chen Y, Liu B, O'Connor JM, et al. Characterization of scatter in cone-beam CT breast imaging: Comparison of experimental measurements and Monte Carlo simulation. Med Phys. 2009; 36(3): 857-69. PMid:19378746.

http://dx.doi.org/10.1118/1.3077122

Published by Sciedu Press 
[12] Henry LJ, Rosenthal MS. Monte Carlo simulation of scatter in non-uniform symmetrical attenuating media for point and distributed sources. Applied Radiation and Isotopes. 1992; 43(3): 449-54. http://dx.doi.org/10.1016/0883-2889(92)90120-4

[13] Ljungberg M, Strand SE, Rajeevan N, et al. Monte Carlo simulation of transmission studies using a planar source with a parallel collimator and a line source with a fan-beam collimator. Nuclear Science, IEEE Transactions on. 1994; 41(4): 1577-84. http://dx.doi.org/10.1109/23.322952

[14] Poludniowski G, Evans PM, Hansen VN, et al. An efficient Monte Carlo-based algorithm for scatter correction in keV cone-beam CT. Physics in Medicine and Biology. 2009; 54(12): 3847-64. PMid:19491449. http://dx.doi.org/10.1088/0031-9155/54/12/016

[15] Yoshizumi MT, Yoriyaz H, Caldas LVE. Backscattered radiation into a transmission ionization chamber: Measurement and Monte Carlo simulation. Applied Radiation and Isotopes. 2010. http://dx.doi.org/10.1016/j.apradiso.2009.10.015

[16] Ioppolo JL, Price RI, Tuchyna T, et al. Diagnostic X-ray dosimetry using Monte Carlo simulation. Phys. Med. Biol. 2002; 47: 1707-20. PMid:12069088. http://dx.doi.org/10.1088/0031-9155/47/10/307

[17] De Oliveira PMC, Squair PL, Lacerda MA, et al. Assessment of organ absorbed doses in patients undergoing chest X-ray examinations by Monte Carlo based softwares and phantom dosimetry. Radiat Measur. 2011; 46(12): 2073-6. http://dx.doi.org/10.1016/j.radmeas.2011.06.058

[18] Boone JM, Seibert JA. Monte Carlo simulation of the scattered radiation distribution in diagnostic radiology. Medical Physics. 1988; 15(5). http://dx.doi.org/10.1118/1.596185

[19] McVey G, Sandborg M, Dance DR, et al. A study and optimization of lumbar spine X-ray imaging systems. The British Journal of Radiology. 2003; 76: 177-88. PMid:12684233. http://dx.doi.org/10.1259/bjr/52734084

[20] Marshall NW, Faulkner K, Warren H. Measured scattered X-ray energy spectra for simulated irradiation geometries in diagnostic radiography. Med. Phys. 1996; 23(7):1271-6. PMid:8839423. http://dx.doi.org/10.1118/1.597690

[21] International Atomic Energy Agency Dosimetry in diagnostic radiology: an international code of practice. Vienna: International Atomic Energy Agency. 2007.

[22] Steinmeyer PR. Ion Chambers: Everything you've wanted to know. RSO Magazine. 2003; 8(5): 11-21.

[23] Ciraj-Bjelac O, Aranđić D, Košutić D, et al. An assessment of scattered radiation during fluoroscopic procedures in diagnostic radioalogy. Nuclear Technology \& Radiation Protection. 2009; 24(3): 204-7. http://dx.doi.org/10.2298/NTRP0903204C

[24] Guillemin M, Gillam L. Ethics, reflexivity, and "ethically important moments" in Research. Qualitative Inquiry. $2004 ; 10: 261$. http://dx.doi.org/10.1177/1077800403262360

[25] Portney LG, Watkins MP. Foundations of Clinical Research: Application to Practice. Prentice Hall. 2009 3rd ed.

[26] Schuster EF. Buffon's needle experiment. The American Mathematical Monthly. 1974 Jan; 81(1). http://dx.doi.org/10.2307/2318907

[27] Lewis RD, Ryde SJ, Hancock DA, et al. A MCNP based model of a linear accelerator X-ray beam. Phys. Med. Biol. 1999; 44: 1219-30. PMid:10368014. http://dx.doi.org/10.1088/0031-9155/44/5/010

[28] Boone JM, Buonocore MH, Cooper VN. Monte Carlo validation in diagnostic radiological imaging. Med. Phys. 2000; 27(6): 1294-1304. PMid:10902559. http://dx.doi.org/10.1118/1.599007

[29] Lazos D, Bliznakova K, Kolitsi Z, et al. An integrated research tool for X-ray imaging simulation. Computer Methods and Programs in Biomedicine. 2002; 70 (3): 241-51. http://dx.doi.org/10.1016/S0169-2607(02)00015-9

[30] Sandborg M, Dance DR, Persliden J, et al. A Monte Carlo Program for the calculation of contrast, noise and absorbed dose in diagnostic radiology. Computer Methods and Programs in Biomedicine. 1994; 42 (3): 167-80. http://dx.doi.org/10.1016/0169-2607(94)90127-9

[31] Zhang GG, Rogers DW, Cygler JE, et al. Monte Carlo investigation of electron beam output factors versus size of square cutout. Med. Phys. 1999; 26(5): 743-50. PMid:10360536. http://dx.doi.org/10.1118/1.598582

[32] Wagner M, Duwenkamp C, Dresing K, et al. An approach to calculate and visualize intraoperative scattered radiation exposure. Stud Health Technol Inform. 2009; 150: 831-5. PMid:19745429.

[33] Hohmann SG, Baeumer C, Bredno J, et al. Simulation and visualization of scattered radiation. US Patent Application Publication. 2010.

[34] Shapiro J. Radiation Protection: A guide for scientists, regulators and physicians. La Editorial, UPR, 2002. 4th ed.

[35] Kawrakow I, Mainegra-Hing E, Rogers DWO, et al. The EGSnrc Code System: Monte Carlo Simulation of Electron and Photon Transport. NRCC Internal Rep. 2013.

[36] Nigapruke K, Puwanich P, Phaisanittisakul N, et al. A comparison of mammographic X-ray spectra: Simulation with EGSnrc and Experiment with CdTe Detector. J. Radiat. Res. 2009; 50: 507-12. http://dx.doi.org/10.1269/jrr.09026

[37] Walters B, Kawrakow I, Rogers DWO. DOSXYZ User's Manual. NRCC Internal Rep. PIRS-0794 (B) (2011).

[38] McVey G, Weatherburn H. A study of scatter in diagnostic X-ray rooms. The British Journal of Radiology. $2004 ; 77: 28-38$. PMid:14988135. http://dx.doi.org/10.1259/bjr/93969091 